\title{
The role of associating liver partition and portal vein ligation for staged hepatectomy in the management of patients with colorectal liver metastasis
}

\author{
Juan Glinka, Victoria Ardiles, Juan Pekolj, Eduardo de Santibañes, Martin de Santibañes \\ Department of General Surgery, Hepato-Bilio-Pancreatic \& Liver Transplantation Unit Hospital Italiano de Buenos Aires, BuenosAires, Argentina \\ Contributions: (I) Conception and design: All authors; (II) Administrative support: None; (III) Provision of study materials or patients: None; (IV) \\ Collection and assembly of data: None; (V) Data analysis and interpretation: J Glinka, M de Santibañes; (VI) Manuscript writing: All authors; (VII) \\ Final approval of manuscript: All authors. \\ Correspondence to: Juan Glinka, MD. Department of Surgery, Division of HPB Surgery, Liver, Transplant Unit, Hospital Italiano de Buenos Aires, \\ Juan D. Perón 4190, C1181ACH, Buenos Aires, Argentina. Email: juan.glinka@hospitalitaliano.org.ar.
}

\begin{abstract}
Associating liver partition and portal vein ligation for staged hepatectomy (ALPPS) approach emerged as a promising surgical strategy for rapid and large hypertrophy of the future liver remnant (FLR) when a major liver resection is necessary. Colorectal liver metastasis (CRLM) is their main indication. However, the promising results published so far, are very difficult to interpret since they usually focus on the technique and not on the underlying disease. Moreover, they are usually made up of complex populations, which received different chemotherapy schemes, with the ALPPS technical variations implemented over time and without consistent long-term follow-up results as well. Whereby, its role in CRLM should be analyzed as carefully as possible to indicate and select the best candidates who will benefit the most from this approach. We conducted a computerized search using PubMed and Google Scholar for reports published so far, using mesh headings and keywords related to the ALPPS and CRLM.
\end{abstract}

Keywords: Liver resection; associating liver partition and portal vein ligation for staged hepatectomy (ALPPS); colorectal cancer; metastasis; liver regeneration

Submitted May 21, 2019. Accepted for publication Aug 06, 2019.

doi: 10.21037/hbsn.2019.08.03

View this article at: http://dx.doi.org/10.21037/hbsn.2019.08.03

\section{Introduction}

Colorectal cancer (CRC) is the third cause of cancer death (1). Colorectal liver metastases (CRLM) are present in $15 \%$ to $25 \%$ of patients at the time of diagnosis; and $50-70 \%$ develop liver metastases during the course of the disease. Unfortunately, a high proportion of patients are not resectable at diagnosis.

In the last decade, advances in systemic chemotherapy, interventional radiology and new surgical strategies, have allowed liver resections with curative intent, in patients where a surgical option was unthinkable. Liver resectability, evolved from the "Ekberg criteria" (2), which focused on what was to be resected, to modern surgical principles that aims to obtain negative free margins of resection with the focus in the sufficient volume/reserve of the future liver remnant (FLR) (3). The knowledge of liver functional reserve is essential because posthepatectomy liver failure (PHLF) is an important cause of mortality after major liver resection (4). Many surgical strategies have been described to induce FLR hypertrophy and prevent PHLF. Most recently, associated liver partition and portal vein ligation for staged hepatectomy (ALPPS) has been presented as a strategy to avoid PHLF by stimulating a rapid and large FLR volume increase. We aim to review the role of ALPPS in the management of patients with CRLM. 


\section{Patient selection and preoperative evaluation}

Since the arrival of ALPPS, promising results have been reported in terms of extraordinary FLR increase. However, its significant high complication rates, created great concern about its safety.

Many studies have been developed to explain this phenomenon. Among them, an inadequate patient selection was overriding in the high morbidity and mortality rates reported in this population so far (5-8).

This concern has been reflected in the recent analysis from the International ALPPS Registry, where 437 patients from 16 centers were included, where a shift in indications toward CRLM after neoadjuvant chemotherapy, from 53\% to $77 \%$, and a reverse trend in biliary tumors, from $24 \%$ to $9 \%$, were observed. With the special emphasis on patients younger than 60 years, with CRLM as the most likely benefited from this surgical strategy $(6,9)$.

The initial candidates for ALPPS in patients with CRLM, should be patients with uni or bilateral disease that are not primarily resectable without a safe FLR.

Conventional approaches such as PVE/PVL will generally be suitable for most patients with extensive CRLM, but when such strategies fail, the ALPPS could be the last potentially curative option for them, and for some authors, its questionless indication (10).

Good quality preoperative imaging is a requisite for good patient selection. Magnetic resonance imaging (MRI) should be performed to investigate small lesions, fatty liver or parenchymal changes after the many chemotherapy schemes that patients with CRLM usually has (11).

Despite this, the hepatic parenchymal quality is usually better when compared to other pathologies that may require an ALPPS, such as hepatocellular carcinoma (HCC) in unhealthy livers or cholestatic biliary tumors. Hence, FLR in CLRM usually has better hypertrophy, regeneration, and functionality, which also explains the better prognosis of these patients.

The positron emission tomography (PET) scan is also recommended to rule out distant disease in these patients who are themselves oncologically borderline.

The KRAS status assessment can be useful as a prognostic indicator and, therefore, selection in patients with CRLM. Recently, it has been shown that patients with KRAS mutation have a significantly worse prognosis in terms of overall survival (OS) and disease-free survival (DFS) after an ALPPS compared to patients with no mutations, or wildtype (WT). Consequently, WT patients would be the most profited from aggressive approaches (12).

From the oncological point of view, the hepatic response to preoperative chemotherapy directed to the CRLM, must be considered also as an important selection parameter, since the better responders to chemotherapy will also be the most benefited from surgery.

Tumor biology can be tested by its response rate to preoperative systemic therapy and thus, have an estimation of the best candidates for ALPPS (13).

Patients who have FLR metastases not amenable to resection, or unresectable extrahepatic disease (including the primary tumor), medical contraindications for liver surgery, elevated anesthetic risk or severe portal hypertension are contraindicated for this type of approach (14). Even though, patients with non-initially resectable CRLM must be treated in multidisciplinary meetings, discussing in a case by case basis, and thus defining, the best therapeutic strategy.

\section{FLR assessment}

The vast majority of fatalities initially from the international registry occurred after the second stage (93\%) and PHLF was the most important cause of death (77\%). Therefore, an adequate assessment of FLR sufficiency before attempting to complete the hepatectomy is key $(15,16)$.

Although the FLR volume is a known predictor of PHLF, and the latter continues to be the most important cause of death in the ALPPS International Registry, the classic volumetric criteria to delimit the adequacy of the FLR at the time of completing the second stage do not seem to be sufficient when applied to a fast-growing liver parenchyma $(17,18)$.

A consensus of experts accepted that the values of cut to proceed with stage 2 are a FLR/total liver volume (FLR/ TLV) $>30-40 \%$, or FLR/BW $>0.5-0.8 \%$ depending on the quality of the FLR. Nevertheless, the reported incidence of PHLF in the International Registry ranged from $16 \%$ to $31 \%$ even when theoretically sufficient FLR volumes were achieved $(19,20)$.

The impressive short-term hypertrophy obtained with ALPPS has led many authors to question the real substrate of this volumetric modification, suggesting that these changes should be interpreted with caution since vascular congestion or even inflammation could interfere in this phenomenon (21). The fact that the vast majority of fatalities initially from the International Registry occurred after the second stage (93\%) and PHLF was the most 

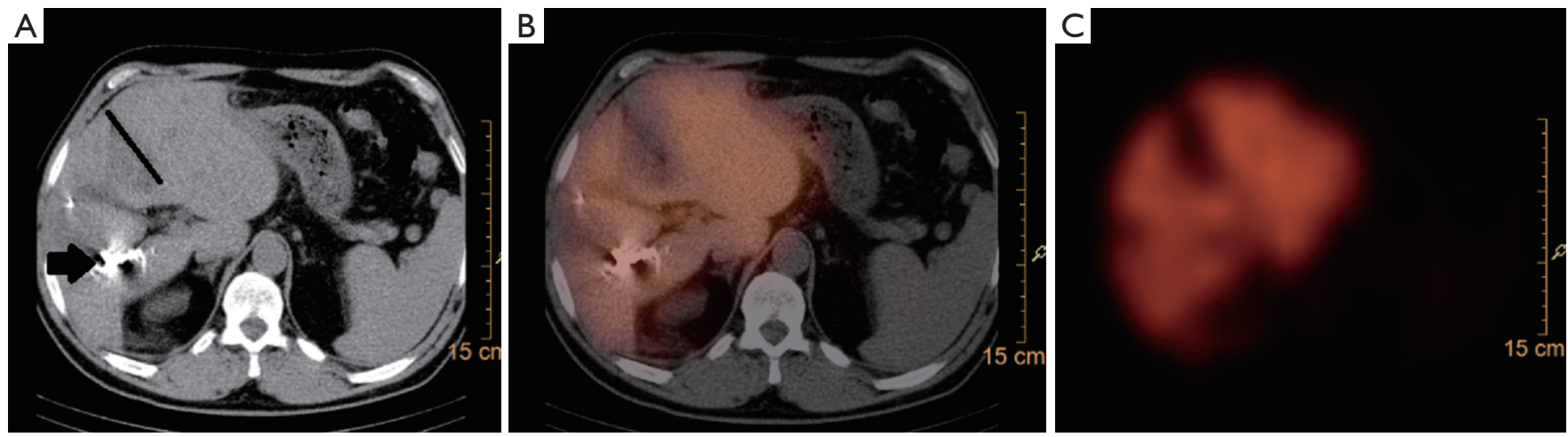

Figure 1 Interstage assessment in associated liver partition and portal vein ligation for staged hepatectomy (ALPPS). (A,B) CT scan-SPECT in the estimation of HIBA index of the FLR in a patient with multiple CRLM after the first stage of mini-ALPPS; (C) the black arrow shows the embolized PV in the disease hemi-liver. The black line shows the limits of the transection line. FLR, future liver remnant; PV, portal vein; CRLM, colorectal liver metastasis.

important cause of death (77\%), clearly indicates that FLR sufficiency defined only by a volumetric criteria is not enough (22).

de Santibañes et al. analyzed the cellular and molecular changes of the hepatocytes associated with the volumetric increase of the liver in patients during ALPPS. Through it, a significantly greater number of hepatocytes were observed in the FLR, as well as the up-regulation of molecular markers of proliferative activity. Those findings indeed demonstrated that quiescent hepatocytes really enter the cell cycle and replicate during ALPPS (23).

However, there is a clear difference in liver function in relation to volume in ALPPS patients due to mechanisms that have not been well clarified yet.

In a recent study to evaluate the relation between FLR volume, liver regeneration characteristics and restoration of function in an experimental model of ALPPS, a clear discordance between the FLR volume increase and functional restoration after ALPPS was observed (24). That is why the modern evaluation of the FLR should focus always on techniques of liver functionality evaluation instead of considering just the volume, and thus, ALPPS should not be performed without functional assessment (25).

The liver function assessment has been evolving historically since the year 1992 when Ekman et al. reported their experience in the measurement of liver uptake function by N 2,6 diethyl-3-iodophenyl carbamoyl iminodiacetic acid (IODIDA) clearance rate (26). On this basis, many years later, the Academic Medical Center (AMC) group in Amsterdam has developed the use of ${ }^{99 \mathrm{~m}} \mathrm{Tc}$ Dynamic-mebrofenin hepatobiliary scintigraphy (HBS) as a quantitative method for evaluating total and regional liver function (including FRL).

The hepatic uptake of ${ }^{99 \mathrm{~m}} \mathrm{Tc}-\mathrm{mebrofenin}$ is similar to the uptake of other organic anions with hepatocyte metabolism such as bilirubin. After its uptake, this labeled compound is excreted into the bile canaliculus without undergoing biotransformation during its transit in the hepatocyte, comparable to other endogenous substances that are metabolized in the liver. This potential, when measured, predicts the functionality of the liver cell. The group that described it, established a value of $2.69 \% / \mathrm{min} / \mathrm{m}^{2}$ of marker excretion as a safety cut-off point to predict PHLF in patients submitted to major hepatectomy regardless of the presence of parenchymal disease (27).

In 2012, as part of a concerted effort to improve ALPPS safety, Serenari et al. have developed a novel parameter consisting of HBS with ${ }^{99 \mathrm{~m}} \mathrm{Tc}-\mathrm{mebrofenin}$ using dynamic planar acquisitions function in addition to a single photon emission CT (SPECT). With this technique-denominated as Hospital Italiano de Buenos Aires (HIBA) Index-the authors have shown that the risk of PHLF in patients with cutoff $<15 \%$ was $80 \%$, whereas no patient with cutoff $>15 \%$ developed PHLF (28). This improves in the measurement accuracy of the regional distribution of liver function represented a revolution in the prevention of PHLF in ALPPS patients (29) (Figure 1).

Given its results and that it would seem to be the only thing that offers concretely to safety profile so far, its systematic use is in candidates for ALPPS is highly recommended.

A relevant aspect to contemplate is that diverse reports 
confirmed that stimulation of the FLR hypertrophy induced by up-regulation of cytokines accelerates tumor growth after PVE and-or PVL (30,31). However, its role in the ALPPS patients-which have per se high recurrence rates-remains unclear. Theoretically, the rapid FLR hypertrophy caused by ALPPS could also be correlated with an accelerated tumor progression. However, recent studies like the carried out by Oldhafer et al., suggests that patients with CRLM may have similar oncological results in the short term compared with traditional methods. Therefore, it seems that the potential for tumor progression in PVE might be at least of the same extent after ALPPS $(32,33)$.

Nevertheless, as there is no current substantial evidence, this perspective must be acknowledged in further investigations to draw comprehensive conclusions

\section{Technical aspects}

In general terms, the ALPPS approach is a two-stage procedure.

Since it was described, the technique consisted of cleaning the FLR by wedge resections (usually the left lateral segment), followed by dissection of the hepatic pedicle, with the subsequent right portal vein ligation (PVL) to later complete the procedure with the parenchymal transection as length of the falciform ligament (in order to interrupt the collateral branches of segment 4) (34).

After 7-14 days, according to the growth of the FLR by CT-volumetry, the second stage is usually completed with right trisectionectomy.

In colorectal cancer, when the primary disease has not been resected previously, the first stage is often associated with the respective colectomy (35).

As a developing technique, many variations to the classical ALPPS were described in order to reduce its invasiveness and, therefore, reduce its morbidity.

Robles et al. have replaced transection during the 1st stage by applying a tourniquet around a parenchymal groove of $1 \mathrm{~cm}$ in the future transection line (tourniquet ALPPS). However, the $64 \%$ morbidity and $9 \%$ mortality in their series did not reflect a real improvement in terms of patient safety (36).

Other authors have proposed to replace parenchymal transection by using radiofrequency (radiofrequency ALPPS) to create a functional liver partition through a "necrotic groove". These approaches resulted in similar hypertrophic profile than the standard ALPPS in addition to fewer complications and mortality rates (37).
Following the trend to less invasiveness, and in order to avoid two major interventions that a classic ALPPS implies, de Santibañes et al. described the "Mini-ALPPS" (38). With this approach, dissection of the pedicle to divide the right $\mathrm{PV}$ is avoided, and with it, the adhesions that hinder the dissection of the hepatic hilum in the second stage. In opposition, an intraoperative and endovascular portal vein embolization (PVE) is performed (Figure 2).

The depth of liver transection is also reduced to significantly decrease the morbidity associated with the procedure without negatively impacting hypertrophy. The current recommendation is not to exceed $3-5 \mathrm{~cm}$, in order to avoid injuring the middle hepatic vein and the vasculobiliary branches of segment 4 . It offers comparable FLR hypertrophy, but significantly lower morbidity, when compared with total transection $(38.1 \%$ vs. $88.9 \% ; \mathrm{P}=0.049)$ and near zero mortality (39).

If there is a CRLM in relation to the transection line in segment 4 , the parenchymal division must be adjusted in order to prevent a tumor invasion of the FLR between the stages.

Then, the second time is completed just like the classic ALPPS.

This approach inverts the conventional ALPPS strategy, minimizing the first stage impact to promote rapid patient recovery and leaving the main surgical procedure for the second stage (Figure 3).

The "Anterior Approach" in ALPPS was an interesting aspect introduced to avoid hepatic hilum manipulation and thus, minimize the surgical impact of the first stage. Besides, avoiding the mobilization of a liver with a great tumor burden, in theory, the risk of hematogenous spread of the disease may also be reduced (40).

Other minimally-invasive technical variants as the totally laparoscopic mini-alpps have been described, but there are no reported data to allow comparisons between the different methods so far (41-44). However, Linecker et al. demonstrated through a multivariate regression analysis that less invasive ALPPS variants were associated with decreased 90-day mortality rates $(\mathrm{P}=0.019)(9)$.

Other non-surgical strategies to increase noticeably and rapidly the FLR are described, such as extended liver venous deprivation-eLVD—by Guiu et al. This novel technique consists of a combination of right $\mathrm{PV}$ embolization and right and middle hepatic vein embolization, where rates of rapid hypertrophy comparable to ALPPS can be achieved, within an amazing safety profile (45). However, patients with CRLM are most likely to have a metastatic bilobar 

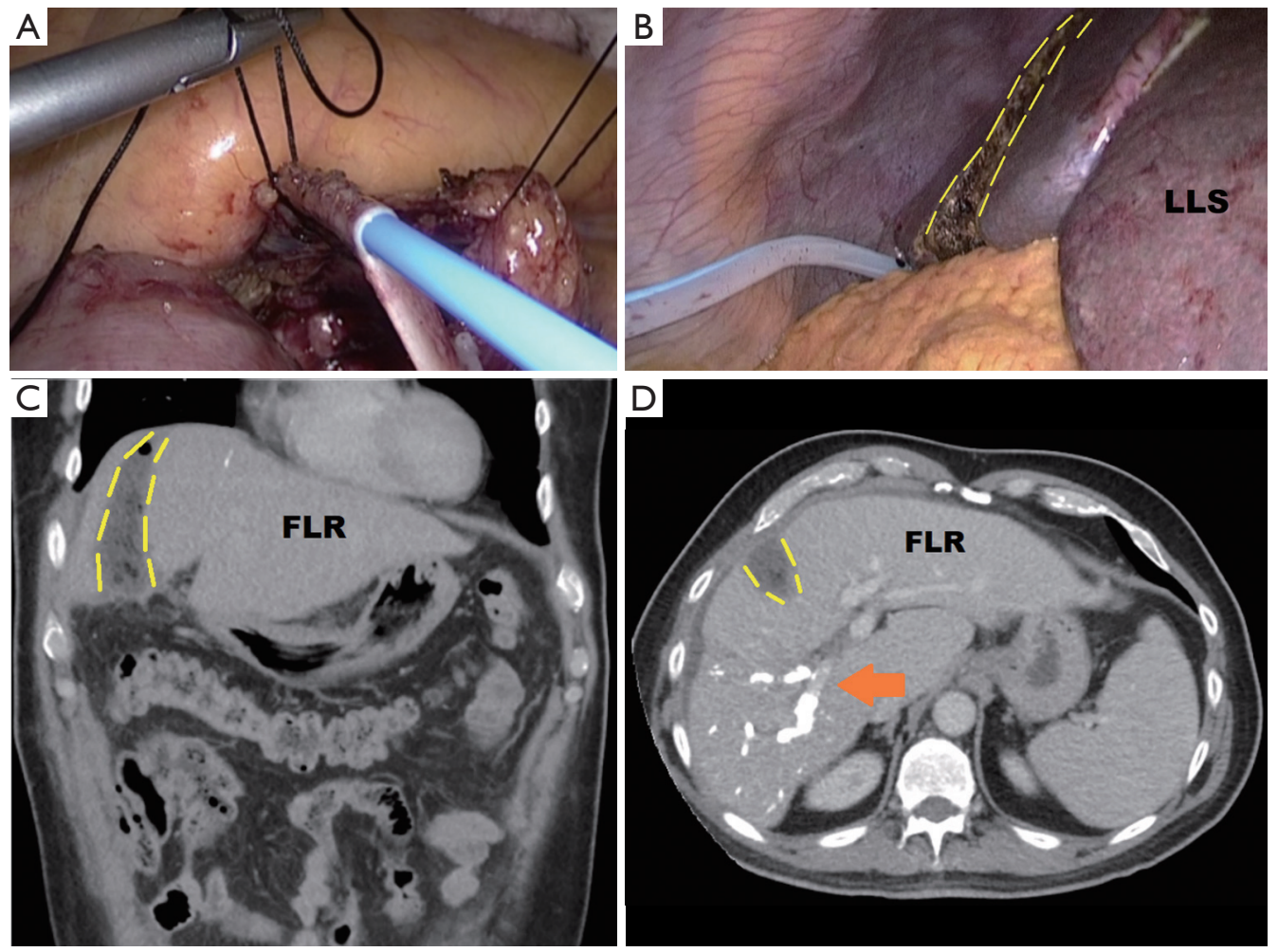

Figure 2 Laparoscopic mini-ALPPS. (A) Laparoscopic catheterization of the IMV with the aim of embolizing the right branch of the PV; (B) laparoscopic sight after the liver surface has been partially transected during the first stage; (C) coronal projection of abdominal CT-scan between the two stages; (D) axial CT-scan projection between the two stages. The transection line is marked with yellow dashed lines. The orange arrow indicates right PV embolization. IMV, inferior mesenteric vein; PV, vein portal; FLR, future liver remnant; LLS, left lateral segment.

disease. Therefore, an important advantage of ALPPS is its possibility of performing large cleanings (usually by atypical resections) of the FLR - even leaving FLR compounded of one segment only-which is entirely necessary before any maneuver that induces the FLR hypertrophy $(46,47)$.

\section{Feasibility of the ALPPS}

Accelerated hypertrophy in ALPPS reduces drop-out in patients with marginally resectable disease, compared to classic two-stage hepatectomy (TSH), where up to $32 \%$ of patients fail to complete tumor resection due to disease progression between stages or insufficient hypertrophy of the FLR (48).

Age greater than 70 years, male sex, tumor larger than $5 \mathrm{~cm}$, a preoperative serum carcinoembryonic antigen level greater than $200 \mathrm{ng} / \mathrm{mL}, 3$ or more metastases in FLR, progression during chemotherapy and the presence of extrahepatic disease have been identified as risk factors for failure to complete the hepatectomy in both uni and multivariate analyses (49).

Schadde et al. published recently a multicentric work that demonstrates that ALPPS is more effective in terms of tumor resection compared to hepatectomy with portal vein emboliztion PVE or PVL ( $83 \%$ vs. 66\%, $\mathrm{P}=0.027$ ) (50). In concordance, other authors have found similar results when comparing ALPPS with TSH (51).

Preliminary results of a prospective randomized protocol of ALPPS versus TSH have recently been published. In this study, 100 patients with comparable baseline characteristics with advanced CLRM, and without another treatment alternative, were randomized to one of both procedures. They concluded that ALPPS was associated with greater resectability than TSH (92\% vs. 57\%) with a similar rate of severe complications, mortality and free margins in the resection (52). However, it is not yet clear if the greater resectability of ALPPS translates later into a survival benefit compared to conventional approaches. 


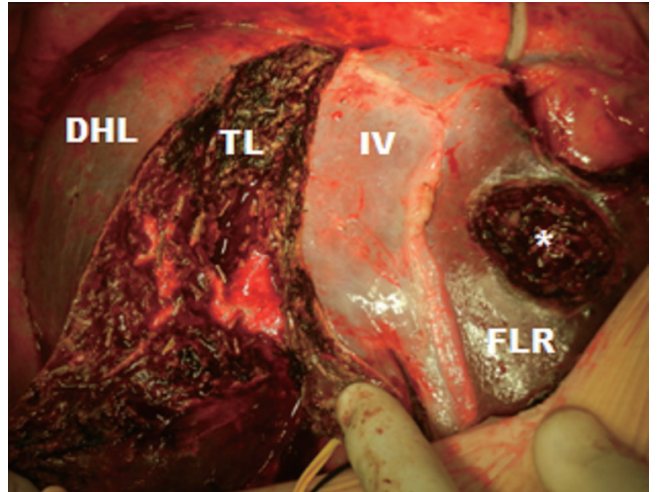

Figure 3 The result after the first stage of the mini-ALPPS approach in a patient with bilobar CRLM. Metastasectomy in the FLR is marked with an asterisk. DHL, disease hemi-liver; TL, transection line; FLR, future liver remnant; IV, segment 4.

\section{Safety of ALPPS: morbidity and mortality}

After being described in Germany, the accelerated global spread of the ALPPS approach was followed by reports of high morbidity and mortality rates (53-56). In the metaanalysis of Schadde et al., 295 patients undergoing this procedure (for primary tumors of different types) were included, reporting a 90 -day mortality of $11 \%$ and $>$ IIIa (Dindo-Clavien) morbidity rate of 44\% (57). However, in the most recent data from the International ALPPS registry considering 528 patients, more acceptable results were obtained, with a mortality rate at 90 days of $8.9 \%$. These results improvements are thanks to the growing evidence worldwide, that allows improvements in the surgical technique as in-patient selection as well.

Considering tumor origin, CLRM has shown the highest safety profile, with $>$ IIIIa morbidity rate of $29 \%$ and mortality at 90 days of $5 \%$ in 228 patients (58).

In addition, a recent multicenter Scandinavian study, as well as a prospective single-center study, have shown that ALPPS can be performed safely in specialized centers, reporting overall mortality rates of $2.8 \%$ and $6.6 \%$ respectively. Remarkably, in the mentioned study, no mortality was observed in the 19 patients with CLRM $(32,59)$. These results are in line with a recent report of Wanis et al. who has reported $21 \%$ morbidity and $0 \%$ mortality in 47 patients with CRLM who underwent an ALPPS for CRLM (60).

Recent data from the International ALPPS Registry indicate that patients who develop PHLF after stage 1 or have a MELD score $>10$ before stage 2 higher risk for 90-day mortality after stage-2 with an odds ratio (OR) 3.9 [95\% confidence interval $(\mathrm{CI}), 1.4-10.9 ; \mathrm{P}=0.01]$ and $\mathrm{OR} 4.9$ (95\% CI, 1.9-12.7, P=0.006), respectively (19).

In addition, age, biliary tumors, operative time of more than $5 \mathrm{~h}$ during stage 1 and the administration of red blood cell transfusions in each stage were identified as significant risk factors for serious complications and mortality at 90 days in ALPPS (6).

On the other hand, a recent prospective study at the Hospital Italiano de Buenos Aires found that the total transection of the parenchyma was an independent risk factor for postoperative complications (39).

The supporters of PVE holds that the ALPPS has excessively high morbidity and mortality rates. However, the recently reported morbidity of $84 \%$ and the $10 \%$ mortality of 87 patients who underwent a major hepatectomy after a PVE or PVL at the Beaujon Hospital in France for the treatment of initially unresectable CRLM does not seem to fully support such assertion (61).

On the other hand, a recent publication with the aim to compare a highly selected series of PVE (78\% as one-stage hepatectomy) with the German multicentric experience of ALPPS, could not confirm a significant difference in the overall morbidity and mortality between both methods $(52,62)$.

Despite efforts to compare ALPPS with PVE, this does not seem entirely correct since both strategies should be used in different scenarios. CRLM represents the main indication for ALPPS in most series, where up to $80 \%$ of treated patients have bilateral disease (39). Since PVE in a one-stage hepatectomy strategy should not be performed in this setting, it appears that the most reasonable and fair comparison of ALPPS is with other two-stage procedures rather than with PVE alone. Considering this, the results obtained from a recent series of ALPPS involving only patients with CLRM are better than the initial series of ALPPS and can be positively compared with most series of two-stage hepatectomies $(39,63)$.

In a recent publication by the MD Anderson Group, among 65 patients who underwent a TSH, 49\% morbidity and $6.4 \%$ mortality were reported considering only the second stage (64). In the experience published in the Paul Brousse Hospital with TSH including 59 patients, morbidity of $59 \%$ and a mortality of $7 \%$ after the second stage was reported (65). In addition, a cooperative experience including 45 patients from two main hepatobiliary centers reported an overall mortality of $8.8 \%$ (4\% after the first stage and $5 \%$ after the second stage) (66). As noted above, 
based on the available data on the most important series of TSH, the reported morbidity and mortality rates are similar to those obtained with the ALPPS approach in recent series.

Finally, it should not be forgotten that the mortality by TSH proposed by the Paul Brousse group was reduced from $15 \%$ in the inaugural series of the year 2000 to $7 \%$ in 2008 and to $4 \%$ in $2014(67,68)$. Therefore, as in any new development, the results of ALPPS will improve while more experience is gained, patient selection is improved, and technical advances are achieved as well.

In a recent publication of the ALPPS registry in 2016 a continuous drop in risk-adjusted early mortality and morbidity was reported, with confirmatory results that patients with CRLM have the lowest complication and mortality rates, even after chemotherapy.

Huiskens et al. have developed a tumor specific-risk score for CRLM showing that older patients with small remnant livers that underwent ALPPS in inexperienced centers, especially after experiencing morbidity after stage- 1 have the worst outcomes (69).

So, this milestone advocates that correct patient selection is the most important factor in preventing serious complications.

\section{Oncological results}

Most of the ALPPS series to date only reported short-term results.

The promising short-term results obtained so far are complex to evaluate oncologically speaking, due to the heterogeneity of the patients, with different underlying pathologies, with variable chemotherapy regimens and the technical variations that have been described and implemented in the published series.

Regarding tumor resectability, there is already strong evidence indicating that ALPPS has higher resectability rates compared to PVE or PVL in classical TSH $(6,39,63)$. The first report of the International ALPPS Registry revealed that both stages of ALPPS could be completed in $98 \%(197 / 202)$ of the patients and comparable to $97 \%$ reported in a meta-analysis that included six studies with 295 patients. The R0 resection rate of the latter was $91 \%$ $(6,57)$. These results contrast the systematic review by Lam et al. 36 in which 459 patients undergoing TSH were analyzed. In this work, the R0 resection was only achieved in $75 \%$ of the patients who finally reached the second stage (48). In addition, in a recent multicenter comparative experience, it has been reported that the rates of complete tumor resection with ALPPS compared to the PVE/PVL was significant $(83 \%$ vs. $66 \%, \mathrm{P}=0.027)$. Although with PVE/PVL, up to $40 \%$ of patients do not reach the second stage, when with ALPPS a complete resection of the tumor in almost all patients is possible (50). This technique offers sufficient hypertrophy in frequently all patients in a short interval and, by splitting the liver, the direct tumor infiltration in the FLR could be avoided.

In the recently released LIGRO Trial (Scandinavian multicenter RCT) involving 97 patients, (almost equally divided between ALPPS and TSH group) a resection rate of $92 \%$ vs. $57 \%$, in favor of ALPPS vs. TSH $(\mathrm{P}<0.0001)$ was observed, with no differences neither in postoperative complications nor mortality (52).

Undoubtedly, the ALPPS approach offers improvements in liver resectability among other approaches. The important unanswered question is whether this would translate into overall survival (OS) and disease-free survival (DFS).

Although with the available evidence this is still unclear, recent studies suggest that patients with CRLM may have similar oncological results in the short term compared to patients treated with traditional methods $(39,50)$.

A multicenter comparative study showed that tumor recurrence at 12 months occurred at a comparable rate in both groups, with 54\% in ALPPS and 52\% in PVE/PVL (6). Data available from the International ALPPS registry in patients with CRLM an OS at 1 and 2 years of $76 \%$ and $62 \%$, as well as a DFS of $59 \%$ and $41 \%$, were respectively observed (median DFS of 14 months) (63). This median of DFS is more than acceptable compared to the 7.5 median months of DFS reported in the updated experience of Hôpital Paul Brousse in TSH (68). In addition, the CRLM subgroup of the International Registry with age $<60$ years showed even best OS, with $88 \%$ and $74 \%$ at 1 and 2 years respectively (average OS of 24 months).

Although only a short-term follow-up is mostly available, these survival terms (based on intention to treat) are similar, or even better, than those of the few existing series of TSH. It must be also considered, that most reports analyze only the OS of patients who reached the second stage (64).

In addition, $64 \%$ of the 3 -year OS in patients with CRLM from the group led by Hans Schlitt are comfortably comparable with traditional approaches (70).

Regarding furthest follow-up, Wanis et al. performed a prospective analysis with 47 patients treated by CLRM from two pioneer centers in ALPPS exclusively including patients with postoperative follow-up $>6$ months. They did 
not report perioperative mortality, and their complication rate was $21 \%$. At 3 years after ALPPS, the overall survival was $50 \%$, while the disease-free survival was $13 \%$.

In spite of that complication rate, almost $80 \%$ of the patients of these series received adjuvant chemotherapy effectively. This would mitigate the great concern regarding the complication rate of ALPPS and its relation to proposing adjuvant chemotherapy in that population. These results were dramatically different from the reported in the initial studies, where the complication rates were so important that the patients were not able to receive chemotherapy treatment after surgery (60) (Table S1).

With a reduction in the surgical complications, better postoperative recovery of patients could be achieved. Consequently, those patients were found to be more likely to receive systemic treatment, which is to our knowledge, a major reason for the enhanced survival reported in this population.

Finally, when survival is analyzed, it must be considered that results are directly related to patient selection, and if we settle for ALPPS those patients with extensive bilateral liver disease (probably not the ideal candidate but the "true" candidate), the poor results should not be surprising. Therefore, current evidence does not allow any firm conclusion regarding oncological superiority or inferiority of ALPPS compared to classical approaches, and data from randomized controlled trials should be awaited to clarify this important aspect.

\section{Conclusions}

ALPPS is an encouraging surgical option in the treatment of patients with CRLM, otherwise unresectable. It is important that it be considered in special circumstances and not necessarily opposed to other procedures or interventions. With the improvements in technical aspects and selection criteria, a decrease in its complication rates were observed. Therefore, patients with CRLM seem to have better postoperative recovery and may be more likely to receive systemic treatment, which is to our knowledge, a major reason for the enhanced survival reported in recent series. Strategies that minimize the ALPPS impact and its related complications should continue to be investigated. Additional studies including long-term follow-up data to clarify the impact of the benefits of this technique in OS and DFS, over others, are still needed.

\section{Acknowledgments}

Funding: None.

\section{Footnote}

Provenance and Peer Review: This article was commissioned by the Guest Editor (Dr. Timothy Pawlik) for the series "Colorectal Cancer Liver Metastasis" published in Hepatobiliary Surgery and Nutrition. The article was sent for external peer review organized by the editorial office.

Conflicts of Interest: All authors have completed the ICMJE uniform disclosure form (available at http://dx.doi. org/10.21037/hbsn.2019.08.03). The authors have no conflicts of interest to declare.

Ethical Statement: The authors are accountable for all aspects of the work in ensuring that questions related to the accuracy or integrity of any part of the work are appropriately investigated and resolved.

Open Access Statement: This is an Open Access article distributed in accordance with the Creative Commons Attribution-NonCommercial-NoDerivs 4.0 International License (CC BY-NC-ND 4.0), which permits the noncommercial replication and distribution of the article with the strict proviso that no changes or edits are made and the original work is properly cited (including links to both the formal publication through the relevant DOI and the license). See: https://creativecommons.org/licenses/by-nc-nd/4.0/.

\section{References}

1. Siegel RL, Miller KD, Jemal A. Cancer statistics, 2018. CA Cancer J Clin 2018;68:7-30.

2. Ekberg H, Tranberg KG, Andersson R, et al. Determinants of survival in liver resection for colorectal secondaries. Br J Surg 1986;73:727-31.

3. Pawlik TM, Schulick RD, Choti MA. Expanding criteria for resectability of colorectal liver metastases. Oncologist 2008;13:51-64.

4. Kishi Y, Abdalla EK, Chun YS, et al. Three hundred and one consecutive extended right hepatectomies: evaluation of outcome based on systematic liver volumetry. Ann Surg 2009;250:540-8.

5. Schnitzbauer AA, Lang SA, Goessmann H, et al. Right 
Portal Vein Ligation Combined With In Situ Splitting Induces Rapid Left Lateral Liver Lobe Hypertrophy Enabling 2-Staged Extended Right Hepatic Resection in Small-for-Size Settings. Ann Surg 2012;25 5:405.

6. Schadde E, Ardiles V, Robles-Campos R, et al. Early survival and safety of ALPPS: first report of the International ALPPS Registry. Ann Surg 2014;260:829-36; discussion 836-8.

7. Nadalin S, Capobianco I, Li J, et al. Indications and limits for associating liver partition and portal vein ligation for staged hepatectomy (ALPPS). Lessons Learned from 15 cases at a single centre. Z Gastroenterol 2014;52:35-42.

8. Belghiti J, Dokmak S, Schadde E. ALPPS: Innovation for innovation's sake. Surgery 2016;159:1287-8.

9. Linecker M, Björnsson B, Stavrou GA, et al. Risk Adjustment in ALPPS Is Associated With a Dramatic Decrease in Early Mortality and Morbidity. Ann Surg 2017;266:779-86.

10. Al Hasan I, Tun-Abraham ME, Wanis KN, et al. Optimizing associated liver partition and portal vein ligation for staged hepatectomy outcomes: Surgical experience or appropriate patient selection? Can J Surg 2017;60:408-15.

11. Sahani DV, Bajwa MA, Andrabi Y, et al. Current status of imaging and emerging techniques to evaluate liver metastases from colorectal carcinoma. Ann Surg 2014;259:861-72.

12. Serenari M, Alvarez FA, Ardiles V, et al. The ALPPS Approach for Colorectal Liver Metastases: Impact of KRAS Mutation Status in Survival. Dig Surg 2018;35:303-10.

13. de Santibañes M, Boccalatte L, de Santibañes E. A literature review of associating liver partition and portal vein ligation for staged hepatectomy (ALPPS): so far, so good. Updates Surg 2017;69:9-19.

14. Lang H, Baumgart J, Mittler J. Associating Liver Partition and Portal Vein Ligation for Staged Hepatectomy in the Treatment of Colorectal Liver Metastases: Current Scenario. Dig Surg 2018;35:294-302.

15. Tanaka K, Matsuo K, Murakami T, et al. Associating liver partition and portal vein ligation for staged hepatectomy (ALPPS): Short-term outcome, functional changes in the future liver remnant, and tumor growth activity. Eur J Surg Oncol 2015;41:506-12.

16. Schadde E, Raptis DA, Schnitzbauer AA. Prediction of Mortality After ALPPS Stage-1: An Analysis of 320 Patients From the International ALPPS Registry. Ann Surg 2015;262:780-5; discussion 785-6.

17. Rahbari NN, Garden OJ, Padbury R, et al.
Posthepatectomy liver failure: a definition and grading by the International Study Group of Liver Surgery (ISGLS). Surgery 2011;149:713-24.

18. Mise Y, Sakamoto Y, Ishizawa T, et al. A worldwide survey of the current daily practice in liver surgery. Liver Cancer 2013;2:55-66.

19. Truant S, Oberlin O, Sergent G, et al. Remnant liver volume to body weight ratio $\geq 0.5 \%$ : a new cut-off to estimate postoperative risks after extended resection in noncirrhotic liver. J Am Coll Surg 2007;204:22-33.

20. Vauthey JN, Chaoui A, Do KA, et al. Standardized measurement of the future liver remnant prior to extended liver resection: methodology and clinical associations. Surgery 2000;127:512-9.

21. Matsuo K, Murakami T, Kawaguchi D, et al. Histologic features after surgery associating liver partition and portal vein ligation for staged hepatectomy versus those after hepatectomy with portal vein embolization. Surgery 2016;159:1289-98.

22. Schadde E, Raptis DA, Schnitzbauer AA, et al. Prediction of Mortality After ALPPS Stage-1: An Analysis of 320 Patients From the International ALPPS Registry. Ann Surg 2015;262:780-5; discussion 785-6.

23. de Santibañes M, Dietrich A, Alvarez FA, et al. Biological Substrate of the Rapid Volumetric Changes Observed in the Human Liver During the Associating Liver Partition and Portal Vein Ligation for Staged Hepatectomy Approach. J Gastrointest Surg 2016;20:546-53.

24. Shi JH, Hammarström C, Grzyb K, Line PD. Experimental evaluation of liver regeneration patterns and liver function following ALPPS. BJS Open 2017;1:84-96.

25. Kang D, Schadde E. Hypertrophy and Liver Function in ALPPS: Correlation with Morbidity and Mortality. Visc Med 2017;33:426-33.

26. Ekman M, Fjälling M, Holmberg S, et al. IODIDA clearance rate: a method for measuring hepatocyte uptake function. Transplant Proc 1992;24:387-8.

27. de Graaf W, van Lienden KP, Dinant S, et al. Assessment of Future Remnant Liver Function Using Hepatobiliary Scintigraphy in Patients Undergoing Major Liver Resection. J Gastrointest Surg 2010;14:369-78.

28. Serenari M, Collaud C, Alvarez F, et al. Interstage assessment of remnant liver function in ALPPS using hepatobiliary scintigraphy: prediction of posthepatectomy liver failure and introduction of the HIBA index. Ann Surg 2018;267:1141-7.

29. Dinant S, de Graaf W, Verwer BJ, et al. Risk assessment of posthepatectomy liver failure using hepatobiliary 
scintigraphy and CT volumetry. J Nucl Med 2007;48:685-92.

30. Kokudo N, Tada K, Seki M, et al. Proliferative activity of intrahepatic colorectal metastases after preoperative hemihepatic portal vein embolization. Hepatology 2001;34:267-72.

31. Hoekstra LT, van Lienden KP, Doets A, et al. Tumor progression after preoperative portal vein embolization. Ann Surg 2012;256:812-7; discussion 817-8.

32. Oldhafer KJ, Donati M, Jenner RM, et al. ALPPS for patients with colorectal liver metastases: effective liver hypertrophy, but early tumor recurrence. World J Surg 2014;38:1504-9.

33. Donati M, Stavrou GA, Oldhafer KJ. Current position of ALPPS in the surgical landscape of CRLM treatment proposals. World J Gastroenterol 2013;19:6548-54.

34. Alvarez FA, Ardiles V, Claria RS, et al. Associating Liver Partition and Portal Vein Ligation for Staged Hepatectomy (ALPPS): Tips and Tricks. J Gastrointest Surg 2013;17:814-21.

35. Choi YI, Moon HH, Shin DH. Two cases of ALPPS procedure: simultaneous ALPPS and colorectal resection and ALPPS procedure for hepatic malignancy larger than 15 centimeter. Ann Hepatobiliary Pancreat Surg 2017;21:151-6.

36. Robles R, Parrilla P, López-Conesa A, et al. Tourniquet modification of the associating liver partition and portal ligation for staged hepatectomy procedure. Br J Surg 2014;101:1129-34; discussion 1134.

37. Wang Q, Yan J, Feng X, et al. Safety and efficacy of radiofrequency-assisted ALPPS (RALPPS) in patients with cirrhosis-related hepatocellular carcinoma. Int J Hyperthermia 2017;33:846-52.

38. de Santibañes E, Alvarez FA, Ardiles V, et al. Inverting the ALPPS paradigm by minimizing first stage impact: the Mini-ALPPS technique. Langenbecks Arch Surg 2016;401:557-63.

39. Alvarez FA, Ardiles V, de Santibañes M, et al. Associating Liver Partition and Portal Vein Ligation for Staged Hepatectomy Offers High Oncological Feasibility With Adequate Patient Safety: A Prospective Study at a Single Center. Ann Surg 2015;261:723-32.

40. Abbasi A, Rahnemai-Azar AA, Merath K, et al. Role of associating liver partition and portal vein ligation in staged hepatectomy (ALPPS)-strategy for colorectal liver metastases. Transl Gastroenterol Hepatol 2018;3:66.

41. Pekolj J, Alvarez FA, Biagiola D, et al. Totally Laparoscopic Mini-ALPPS Using a Novel Approach of Laparoscopic-
Assisted Transmesenteric Portal Vein Embolization. J

Laparoendosc Adv Surg Tech A 2018;28:1229-33.

42. Chan AC, Pang R, Poon RT. Simplifying the ALPPS procedure by the anterior approach. Ann Surg 2014;260:e3.

43. Lau WY, Lai EC. Modifications of ALPPS - from complex to more complex or from complex to less complex operations. Hepatobiliary Pancreat Dis Int 2017;16:346-52.

44. Machado MAC, Makdissi FF, Surjan RC. Totally laparoscopic ALPPS is feasible and may be worthwhile. Ann Surg 2012;256:e13; author reply e16-9.

45. Guiu B, Quenet F, Escal L, et al. Extended liver venous deprivation before major hepatectomy induces marked and very rapid increase in future liver remnant function. Eur Radiol 2017;27:3343-52.

46. Schadde E, Malagó M, Hernandez-Alejandro R, et al. Monosegment ALPPS hepatectomy: extending resectability by rapid hypertrophy. Surgery 2015;157:676-89.

47. Soggiu F, Giovinazzo F, Straiton J, et al. Monosegment ALPPS hepatectomy preserving segment 4 for colorectal liver metastases: literature review and our experience. Hepatobiliary Surg Nutr 2018;7:105-15.

48. Lam VWT, Laurence JM, Johnston E, et al. A systematic review of two-stage hepatectomy in patients with initially unresectable colorectal liver metastases. HPB 2013;15:483-91.

49. Giuliante F, Ardito F, Ferrero A, et al. Tumor Progression During Preoperative Chemotherapy Predicts Failure to Complete 2-Stage Hepatectomy for Colorectal Liver Metastases: Results of an Italian Multicenter Analysis of 130 Patients. J Am Coll Surg 2014;219:285-94.

50. Schadde E, Ardiles V, Slankamenac K, et al. ALPPS Offers a Better Chance of Complete Resection in Patients with Primarily Unresectable Liver Tumors Compared with Conventional-Staged Hepatectomies: Results of a Multicenter Analysis. World J Surg 2014;38:1510-9.

51. Zhou Z, Xu M, Lin N, et al. Associating liver partition and portal vein ligation for staged hepatectomy versus conventional two-stage hepatectomy: a systematic review and meta-analysis. World J Surg Oncol 2017;15:227.

52. Sandström P, Røsok BI, Sparrelid E, et al. ALPPS Improves Resectability Compared With Conventional Two-stage Hepatectomy in Patients With Advanced Colorectal Liver Metastasis: Results From a Scandinavian Multicenter Randomized Controlled Trial (LIGRO Trial). Ann Surg 2018;267:833-40.

53. de Santibañes E, Ardiles V, Alvarez FA, et al. Extreme Hepatic Surgery and Other Strategies: Increasing 
Resectability in Colorectal Liver Metastases. Cham: Springer; 2017:383.

54. Torres OJ, Fernandes Ede S, Oliveira CV, et al. Associating liver partition and portal vein ligation for staged hepatectomy (ALPPS): the Brazilian experience. Arq Bras Cir Dig 2013;26:40-3.

55. Ratti F, Schadde E, Masetti M, et al. Strategies to Increase the Resectability of Patients with Colorectal Liver Metastases: A Multi-center Case-Match Analysis of ALPPS and Conventional Two-Stage Hepatectomy. Ann Surg Oncol 2015;22:1933-42.

56. Serenari M, Zanello M, Schadde E, et al. Importance of primary indication and liver function between stages: results of a multicenter Italian audit of ALPPS 2012-2014. HPB 2016;18:419-27.

57. Schadde E, Schnitzbauer AA, Tschuor C, et al. Systematic Review and Meta-Analysis of Feasibility, Safety, and Efficacy of a Novel Procedure: Associating Liver Partition and Portal Vein Ligation for Staged Hepatectomy. Ann Surg Oncol 2015;22:3109-20.

58. Linecker M, Stavrou GA, Oldhafer KJ, et al. The ALPPS Risk Score. Ann Surg 2016;264:763-71.

59. Røsok BI, Björnsson B, Sparrelid E, et al. Scandinavian multicenter study on the safety and feasibility of the associating liver partition and portal vein ligation for staged hepatectomy procedure. Surgery 2016;159:1279-86.

60. Wanis KN, Ardiles V, Alvarez FA, et al. Intermediateterm survival and quality of life outcomes in patients with advanced colorectal liver metastases undergoing associating liver partition and portal vein ligation for staged hepatectomy. Surgery 2018;163:691-7.

61. Cauchy F, Aussilhou B, Dokmak S, et al. Reappraisal of the risks and benefits of major liver resection in patients with initially unresectable colorectal liver metastases. Ann Surg 2012;256:746-52; discussion 752-4.

62. Shindoh J, Vauthey JN, Zimmitti G, et al. Analysis of the Efficacy of Portal Vein Embolization for Patients

Cite this article as: Glinka J, Ardiles V, Pekolj J, de Santibañes E, de Santibañes M. The role of associating liver partition and portal vein ligation for staged hepatectomy in the management of patients with colorectal liver metastasis. HepatoBiliary Surg Nutr 2020;9(6):694-704. doi: 10.21037/hbsn.2019.08.03 with Extensive Liver Malignancy and Very Low Future Liver Remnant Volume, Including a Comparison with the Associating Liver Partition with Portal Vein Ligation for Staged Hepatectomy Approach. J Am Coll Surg 2013;217:126-33; discussion 133-4.

63. Hernandez-Alejandro R, Bertens KA, Pineda-Solis $\mathrm{K}$, et al. Can we improve the morbidity and mortality associated with the associating liver partition with portal vein ligation for staged hepatectomy (ALPPS) procedure in the management of colorectal liver metastases? Surgery 2015;157:194-201.

64. Brouquet A, Abdalla EK, Kopetz S, et al. High Survival Rate After Two-Stage Resection of Advanced Colorectal Liver Metastases: Response-Based Selection and Complete Resection Define Outcome. J Clin Oncol 2011;29:1083-90.

65. Wicherts DA, Miller R, de Haas RJ, et al. Long-term results of two-stage hepatectomy for irresectable colorectal cancer liver metastases. Ann Surg 2008;248:994-1005.

66. Tsai S, Marques HP, De Jong MC, et al. Two-stage strategy for patients with extensive bilateral colorectal liver metastases. HPB (Oxford) 2010;12:262-9.

67. Adam R, Laurent A, Azoulay D, et al. Two-stage hepatectomy: A planned strategy to treat irresectable liver tumors. Ann Surg 2000;232:777-85.

68. Faitot F, Faron M, Adam R, et al. Two-Stage Hepatectomy Versus 1-Stage Resection Combined With Radiofrequency for Bilobar Colorectal Metastases: A Case-Matched Analysis of Surgical and Oncological Outcomes. Ann Surg 2014;260:822-7; discussion 827-8.

69. Huiskens J, Schadde E, Lang H, et al. Avoiding postoperative mortality after ALPPS-development of a tumor-specific risk score for colorectal liver metastases. HPB (Oxford) 2019;21:898-905.

70. Lang SA, Loss M, Benseler V, et al. Long-term results after in-situ split (ISS) liver resection. Langenbecks Arch Surg 2015;400:361-9. 